\title{
Is the Rehbein procedure obsolete in the treatment of Hirschsprung's disease?
}

\author{
Reina Visser • Teun J. van de Ven • \\ Iris A. L. M. van Rooij • Rene M. H. Wijnen • \\ Ivo de Blaauw
}

Published online: 24 August 2010

(C) The Author(s) 2010. This article is published with open access at Springerlink.com

\begin{abstract}
Purpose After 25 years of practice and positive results of the Rehbein-procedure (RB) for children with Hirschsprung Disease (HD), we changed to the less invasive transanal endorectal pull through (TERPT). The aim of this study was to compare short- and mid-term complications of these two procedures in our patients with HD.

Methods Retrospective data of 50 HD patients were analyzed. Of these patients, 25 underwent RB (2000-2006) and in 25 the TERPT was performed (2005-2009). Medical records were reviewed to score complications and outcomes. Differences were analyzed using Chi-Square and Mann-Whitney $U$ tests.

Results All RB patients (100\%) were given a colostomy compared with four patients $(16 \%)$ in the TERPT group $(p<0.001)$. The average age at surgery in the RB group was 191 days whereas this was 72 days in the TERPT group $(p<0.01)$. The mean length of time of surgery in the RB group (158 min) was not significantly different from that in the TERPT group (183 min). Ganglion cells were located in all specimens at the proximal end of the
\end{abstract}

R. Visser · T. J. van de Ven · I. de Blaauw ( $₫)$

Department of Surgery/Pediatric Surgery, Radboud University

Nijmegen Medical Center, PO Box 9101,

6500 HB Nijmegen, The Netherlands

e-mail: deblaauw@wxs.nl

I. A. L. M. van Rooij

Department of Epidemiology, Biostatistics and HTA,

Radboud University Nijmegen Medical Center,

Nijmegen, The Netherlands

R. M. H. Wijnen · I. de Blaauw

Department of Paediatric Surgery,

Sophia Children's Hospital-Erasmus MC,

Rotterdam, The Netherlands specimens. The median time to first feeding significantly decreased from 2 days (range 1-11) in the RB group to 1 day (range $1-3$ ) in the TERPT group $(p<0.001)$. The median length of hospital stay decreased in the TERPT group ( 8 days) compared with the RB group (10 days) $(p<0.001)$. There was a significant reduction in postoperative obstructive symptoms during the first 6 months in the TERPT group (48\%) compared with the RB group (84\%) $(p=0.016)$. Postoperative enterocolitis decreased from $40 \%$ in the RB group to $24 \%$ in the TERPT group although this was not statistically significant.

Conclusions The introduction of TERPT reduced the need for colostomies; it shortened days to first feeding after surgery and reduced hospital stay. It also improved shortterm outcome with less obstructive symptoms. We recommend TERPT surgery as a first choice in children with HD. we consider the RB now to be obsolete.

Keywords Rehbein - Transanal endorectal pull through Hirschsprung · Hirschsprung's disease - Retrospective · Obstructive symptoms · Enterocolitis

\section{Introduction}

The Rehbein-procedure (RB) for children with Hirschsprung Disease (HD) is one of the techniques mostly used in Europe for the past 25 years [1, 2]. In our clinic good long-term results were seen despite the fact that a relatively large aganglionotic segment was left in situ compared with other techniques as the Soave, Swenson, or Duhamel procedures [3]. The long-term results showed slightly more constipation after RB but less soiling compared with other techniques. We concluded at that time that the RB was not as obsolete as was suggested by others [3, 4]. 
However, more than a decade ago De la Torre and Langer almost simultaneously described a less invasive technique for HD [5, 6]. The transanal endorectal pull through (TERPT) is a one-stage-procedure (no colostomy required) and it does not require a laparotomy or laparoscopy, but is fully performed transanally. Risks of contamination and adhesion formation are minimal and the procedure does not damage the pelvic structures [7]. In our clinic the TERPT was introduced in 2005. Only a few studies compared older techniques with the more recent TERPT [7-10]. The aim of the present study was to compare short- and mid-term complications and outcome of patients from our University Hospital who underwent the RB or TERPT procedure.

\section{Methods}

All patients with HD who were treated at the Radboud University Nijmegen Medical Centre (RUNMC) in the Netherlands between 2000 until 2009 were included in the study. Concerning pediatric surgery, the RUNMC is a tertiary referral Hospital with a coverage area of approximately 5 million people. In 2004 and 2005 we transitioned from the RB to the TERPT. The medical records of the last consecutive 25 patients who had undergone a RB in the period of 2000-2006 were collected to compare with the first 25 consecutive patients who were treated with a TERPT from 2005 until 2009. Patients who underwent primary surgery at other medical centers were excluded. The following data were retrospectively studied based on the medical records: associated congenital anomalies, age at surgery, weight at surgery, presence of colostomy, details of all surgical procedures (length of surgery, length of resected colon, level of the resection, presence or absence of ganglion cells at the proximal end of the anastomosis, amount of blood loss and blood transfusion), time to first feeding, length of hospital stay, early ( $<30$ days) and late postoperative complications, mortality, episodes of enterocolitis postoperatively, need for laxatives and rectal irrigations and stool habits including continence at the age of 4 years. Enterocolitis was defined as a period of fever, food intolerance with watery, explosive, and smelly diarrhea. Obstructive symptoms are defined as need for dilatations or botuline treatment, prolonged need for laxative treatment or bowel irrigations, or admission for bowel obstruction. All symptoms except for age and weight at surgery, length of aganglionosis, time to first feeding, length of hospital stay, and episodes of enterocolitis were scored as present or absent. Differences between the both treatment groups regarding dichotomous variables were analyzed using Chi-Square test, and regarding continuous parameters Mann-Whitney $U$ test was performed.

\section{Results}

There were 25 patients who underwent RB between 2000 and 2006, and there were 25 patients who underwent TERPT between 2005 and 2009. Complete evaluation was carried out in all 50 cases. Descriptive data of all cases are shown in Table 1. There were no significant differences between the $\mathrm{RB}$ and TERPT group regarding gender and associated congenital anomalies, most often Down syndrome (16 vs. $12 \%$, respectively) and congenital heart anomalies (28 vs. $8 \%$, respectively). Age and weight at time of surgery were significantly decreased in the TERPT group as compared with the RB group (mean age TERPT group 72 days, mean age RB group 191 days, $p=0.006$ ). All RB patients were given a colostomy in comparison to four of the TERPT patients $(p<0.001)$.

Surgical characteristics are given in Table 2. The median length of time of surgery in de RB group (155 min) was
Table 1 General characteristics of Hirschsprung patients treated with Rehbein or TERPT procedure

$n s$ not statistically significant $(p>0.05)$

${ }^{a}$ Median (5-95\% range)

Table 2 Surgical characteristics of Hirschsprung patients treated with the Rehbein or TERPT procedure

$n s$ not statistical significant $(p>0.05)$

${ }^{\mathrm{a}}$ Median (5-95\% range)

\begin{tabular}{|c|c|c|c|}
\hline Characteristics & Rehbein $(n=25)$ & TERPT $(n=25)$ & $p$ value \\
\hline Male:female & $23: 2$ & $20: 5$ & ns \\
\hline Congenital anomalies (\%) & $8(32)$ & $5(20)$ & $\mathrm{ns}$ \\
\hline Colostomy (\%) & $25(100)$ & $4(16)$ & $<0.001$ \\
\hline Age at surgery in days ${ }^{\mathrm{a}}$ & $191(68-2,278)$ & $72(18-3,637)$ & $<0.01$ \\
\hline Weight at surgery in grams $\mathrm{s}^{\mathrm{a}}$ & $6,990(5,399-21,250)$ & $5,360(3,255-32,450)$ & 0.01 \\
\hline
\end{tabular}

\begin{tabular}{|c|c|c|c|}
\hline & Rehbein $(n=25)$ & TERPT $(n=25)$ & $p$ value \\
\hline Proximal end ganglionic cells (\%) & $25(100)$ & $25(100)$ & $\mathrm{ns}$ \\
\hline Operating time $(\min )^{\mathrm{a}}$ & $155(68-256)$ & $154(108-317)$ & ns \\
\hline Length of resection $(\mathrm{cm})^{\mathrm{a}}$ & $15(8-36)$ & $25(15-50)$ & $<0.001$ \\
\hline Time to first feeding (days) ${ }^{a}$ & $2(1-10)$ & $1(1-3)$ & $<0.001$ \\
\hline Length of hospital stay (days) $)^{a}$ & $8(5-45)$ & $6(3-25)$ & 0.01 \\
\hline
\end{tabular}


not different from the TERPT group (154 min). During both the RB and TERPT, ganglion cells were located in all specimens at the proximal end of the anastomosis. The median length of the resected bowel was $15 \mathrm{~cm}$ in the RB group and $25 \mathrm{~cm}$ in the TERPT group. Intra operative blood loss was similar in both groups and no transfusions were needed in both groups. The median time to first feeding significantly decreased from 2 to 1 day when we compared the RB group with the TERPT. Also, the length of hospital stay decreased significantly in the TERPT group (6 days, range $3-25$ ) compared with the RB group (8 days, range 5-45).

There was no mortality in both groups. Early complications ( $<30$ days) are given in Table 3 . Wound infections, early stenosis, leakage, sepsis, and relaparotomy were rare and not different between the two groups. There were no different episodes of enterocolitis seen in the RB group $(n=1)$ compared with the TERPT group $(n=1)$.

However, in the follow-up of the first 6 months significant changes were observed (Table 4). There were more obstructive symptoms in the RB group than in the TERPT group $(p=0.016)$. In the $\mathrm{RB}$ group 20 children needed admission for bowel obstruction (versus 12 children in the TERPT group), 5 children underwent botuline treatment (compared with 4 children in the TERPT group), 15 children had a prolonged need for laxatives (versus 9 children in the TERPT group) and 7 children needed colon irrigations (compared with 9 children in the TERPT group). In the first 6 months episodes of enterocolitis occurred in $40 \%$ of the

Table 3 Early complications ( $<30$ days) after the Rehbein and TERPT procedure

\begin{tabular}{llll}
\hline Complications & Rehbein, $n(\%)$ & TERPT, $n(\%)$ & $p$ value \\
\hline Wound infection & $2(8)$ & 0 & $\mathrm{~ns}$ \\
Leakage & $4(16)$ & $1(4)$ & $\mathrm{ns}$ \\
Stenosis & $1(4)$ & 0 & $\mathrm{~ns}$ \\
Ileus & 0 & $1(4)$ & $\mathrm{ns}$ \\
Pneumonia & 0 & 0 & $\mathrm{~ns}$ \\
Sepsis & $1(4)$ & 0 & $\mathrm{~ns}$ \\
Enterocolitis & $1(4)$ & $1(4)$ & $\mathrm{ns}$ \\
Redo laparotomy & $2(8)$ & $3(12)$ & $\mathrm{ns}$ \\
\hline
\end{tabular}

$n s$ not statistical significant $(p>0.05)$

Table 4 Late complications ( $<6$ months) of the Rehbein and TERPT procedure

\begin{tabular}{lcll}
\hline Complications & Rehbein, $n(\%)$ & TERPT, $n(\%)$ & $p$ value \\
\hline Enterocolitis & $10(40)$ & $6(25)$ & $\mathrm{ns}$ \\
Obstructive symptoms & $20(80)$ & $12(50)$ & 0.03 \\
Incisional hernia & $2(8)$ & 0 & $\mathrm{~ns}$
\end{tabular}

$n s$ not statistical significant $(p>0.05)$
RB group and $25 \%$ of the TERPT group but this was not significant. There were two cases with a incisional hernia in the RB group. The follow-up was too short to properly measure fecal continence because most children in the TERPT group have not reached the age of potty training yet.

\section{Discussion}

The transanal endorectal pull through (TERPT) procedure for Hirschsprung's disease has proven to be a simple and effective procedure. It was first described by de la Torre and Langer in the late 1990s [5, 6]. The major advantages suggested are that this procedure is less invasive, less painful, has a shorter time to feeding, has a shorter hospital stay, and gives a better cosmetic outcome with no abdominal scar [11]. However, only a few studies have actually compared the TERPT with previous techniques. Most of our cases treated with TERPT were performed at the age of $8-10$ weeks, by one or two surgeons at a single institute making comparison more valuable. All patients in the TERPT group used rectal irrigation pending surgery, thereby eliminating the need for a colostomy. Because the RB was done at an average of 6 months it used to be preceded by a colostomy. This was part of the protocol in all patients with RB as protection in case of anastomotic leakage. Anastomotic leakage is theoretically less of an issue after the TERPT as the anastomosis is just $1-2 \mathrm{~cm}$ above the dentate line. No colostomy is obviously the first advantage of the TERPT procedure as this rules out all colostomy-related complications. At present, in many centers the TERPT is performed as soon as the diagnosis is made, even in small newborns [12]. We are also considering surgery in the newborn which eliminates the need for parents to start irrigations at home.

Although operating time was not different between the $\mathrm{RB}$ and TERPT procedures, recovery was faster in the TERPT group. This was reflected in the days until first feeding and length of hospital stay. Thus, again in our setting (single institute, same surgeons) the TERPT procedure appears to result in earlier recovery. Furthermore, it is obvious that with no abdominal scars, the TERPT results in excellent cosmetic outcome.

The length of resection was different in both groups. This was not previously described by others. It can be explained by the fact that with the Rehbein technique approximately $2-3 \mathrm{~cm}$ of aganglionotic rectum is left. The difference possibly also reflects the fact that the RB is an open procedure in which the surgeon may have a better view of the transition zone. This may result in smaller resections in the RB group compared with the TERPT group. However, in both groups all specimen had ganglion cells at the proximal resection site and all were appropriate. The leakage rate did not differ significantly between the RB (16\%) and TERPT (4\%) 
groups. For the RB it was slightly higher than in most open studies in which leakage rate varies between 5 and $11 \%$ [13]. However, all were protected by a colostomy. All could be managed with abdominal drainage or conservative treatment only and no anastomosis needed to be dismantled. All healed without a further stricture of the anastomosis.

In the TERPT group the leakage rate was similarly low as in other studies [14-16]. Leakage of the anastomosis is less of a problem as the anastomosis is just above the dentate line. Our case with an anastomotic leakage was managed conservatively for 7 days with good outcome.

There was a significant higher amount of obstructive symptoms (after 30 days) in the RB group compared with the TERPT group ( $p=0.02$ ). Obstructive symptoms occurred in 20 children in the RB group compared with 12 children in the TERPT group. Less burden of obstructive symptoms is in line with previous reports [7]. In line with this we observed a trend towards fewer postoperative episodes of enterocolitis 30 days after surgery in the TERPT group as compared with the RB group. Enterocolitis is often related to postoperative obstructive symptoms. Enterocolitis is considered to be one of the major and most serious complications of Hirschsprung's disease. It occurs mostly preoperatively, but it is also seen postoperatively in approximately $9-17 \%$ cases after TERPT. The variability in literature is most probably related to the use of different criteria to define enterocolitis. Postoperative enterocolitis seems to be related to the age at which patients are operated (more enterocolitis at neonatal age) [17]. However, leaving a shorter muscle cuff has also been suggested to reduce the postoperative enterocolitis rate [12]. We therefore changed our technique the last few years and now only leave a 1-2 cm muscle cuff making it more a Swenson-like procedure. This has been supported and recommended by others [18]. A shorter muscle cuff gives less obstructive symptoms [19] and less episodes of enterocolitis. There were no significant differences in enterocolitis between the two groups in our study. However, comparing the TERPT with a larger series of the Rehbein technique in our hospital which we previously described, the TERPT has less postoperative enterocolitis [3].

Continence can only be evaluated for children who are at least 3 years old. The follow up of our study was too short to properly measure fecal continence.

In conclusion, the introduction of TERPT reduced the need for colostomies; it shortened days to first feeding after surgery and reduced hospital stay. It also improved shortand mid-term outcome with less obstructive symptoms. Finally, no visible scars are seen after TERPT. We recommend TERPT surgery as a first choice in children with HD and consider the RB now to be obsolete.

Open Access This article is distributed under the terms of the Creative Commons Attribution Noncommercial License which permits any noncommercial use, distribution, and reproduction in any medium, provided the original author(s) and source are credited.

\section{References}

1. Holschneider AM (2000) Experiences with Rehbein technique. Harwood, Chichester, pp 366-374

2. Fuchs O, Booss D (1999) Rehbein's procedure for Hirschsprung's disease. An appraisal of 45 years. Eur J Pediatr Surg 9(6):389-391

3. Joosten KFM, Festen C, Staak FHJM (1988) Is Rehbein's operation an obsolete method of treating Hirschsprung's disease. Pediatr Surg Int 3:204-207

4. Hartman EE, Sprangers MA, Visser MR, Oort FJ, Hanneman MJ, van Heurn LW et al (2006) Hirschsprung's disease: healthcare meets the needs. J Pediatr Surg 41(8):1420-1424

5. De La Torre-Mondragón L, Ortega-Salgado JA (1998) Transanal endorectal pull-through for Hirschsprung's disease. J Pediatr Surg 33(8):1283-1286

6. Langer JC, Minkes RK, Mazziotti MV, Skinner MA, Winthrop AL (1999) Transanal one-stage Soave procedure for infants with Hirschsprung's disease. J Pediatr Surg 34(1):148-151

7. El-Sawaf MI, Drongowski RA, Chamberlain JN, Coran AG, Teitelbaum DH (2007) Are the long-term results of the transanal pull-through equal to those of the transabdominal pull-through? A comparison of the 2 approaches for Hirschsprung disease. J Pediatr Surg 42:41-47

8. Aslanabadi S, Ghalehgolab-Behbahan A, Zarrintan S, Jamshidi M, Seyyedhejazi M (2008) Transanal one-stage endorectal pullthrough for Hirschsprung's disease: a comparison with the staged procedures. Pediatr Surg Int 24:925-929

9. De La Torre L, Ortega A (2000) Transanal versus open endorectal pull-through for Hirschsprung's disease. J Pediatr Surg 35(11): $1630-1632$

10. Hadidi A (2003) Transanal endorectal pull-through for Hirschsprung's disease: a comparison with the open technique. Eur J Pediatr Surg 13:176-180

11. Rangel SJ, de Blaauw I (2010) Advances in pediatric colorectal surgical techniques. Semin Pediatr Surg 19:86-95

12. De La Torre L, Langer JC (2010) Transanal endorectal pull-through for Hirschsprung disease: technique, controversies, pearls, pitfalls, and an organized approach to the management of postoperative obstructive symptoms. Semin Pediatr Surg 19:96-106

13. Sherman JO, Snyder ME, Weitzman JJ, Jona JZ, Gillis DA, O'Donnell B et al (1989) A 40-year multinational retrospective study of 880 Swenson procedures. J Pediatr Surg 24:833-838

14. Aslan MK, Karaman I, Karaman A, Erdogan D, Cavusoglu YH, Cakmak O (2007) Our experience with transanal endorectal pullthrough in Hirschsprung's disease. Eur J Pediatr Surg 17:335-339

15. Langer JC, Durrant AC, De la TL, Teitelbaum DH, Minkes RK, Caty MG et al (2003) One-stage transanal Soave pullthrough for Hirschsprung disease: a multicenter experience with 141 children. Ann Surg 238:569-583

16. Hadidi A (2003) Transanal endorectal pull-through for Hirschsprung's disease: experience with 68 patients. J Pediatr Surg 38:1337-1340

17. Elhalaby EA, Hashish A, Elbarbary MM, Soliman HA, Wishahy MK, Elkholy A et al (2004) Transanal one-stage endorectal pullthrough for Hirschsprung's disease: a multicenter study. J Pediatr Surg 39:345-351

18. Rintala RJ (2003) Transanal coloanal pull-through with a short muscular cuff for classic Hirschsprung's disease. Eur J Pediatr Surg 13:181-186

19. Nasr A, Langer JC (2007) Evolution of the technique in the transanal pull-through for Hirschsprung's disease: effect on outcome. J Pediatr Surg 42:36-39 